\title{
Morfologías resilientes: Desde lo digital a la materia
} Resilient morphologies: From digital to matter

\author{
Daniela Frogheri \\ Universidad de Monterrey, México \\ daniela.frogheri@udem.edu \\ Fernando Meneses-Carlos \\ Universidad de Monterrey, México \\ fernando.meneses@udem.edu
}

Alberto T. Estévez

Universitat Internacional de Catalunya, España

estevez@uic.es

\begin{abstract}
This paper presents a research applied to the development of resilient morphologies from the digital environment to its materialization. The work consists in the generation of such morphologies understood as parametric-associative systems, constituted by proliferations of components defined through formal languages, and in the study of their various types of resilience, both in the digital environment and in their physical materialization.
\end{abstract}

Keywords: Morfologías; Resiliencia; Sistemas; Diseño paramétrico-asociativo; Morfogénesis.

\section{Introducción}

En este paper se presenta una investigación aplicada al desarrollo de morfologías resilientes desde el entorno digital hasta su materialización.

El trabajo consiste en la generación de dichas morfologías que se entienden como sistemas paramétrico-asociativos, constituidos por proliferaciones de componentes definidas a través de lenguajes formales, y en el estudio de sus varios tipos de resiliencia, sea en el entorno digital que en su materialización física.

La investigación surge desde algunas consideraciones sobre la actual relevancia del concepto de resiliencia en el campo del diseño en todas sus escalas, con una mirada hacia los procesos de generación de las formas y en especial hacia aquellos que conciernen las geometrías complejas.

Los dos motores principales del trabajo, ambos relacionados con los avances de la tecnología y con su difusión masiva, son la elevada capacidad de comunicación entre entidades similares y diferentes, característica de nuestra época, y la también elevada capacidad de reconocer y manejar la complejidad a través de la cual interpretamos lo existente y generamos nuevos artefactos.

Por lo que concierne la capacidad de comunicación, podemos decir que hoy en día, sobre todo gracias a la universalidad de lo digital en cuanto lenguaje, nos encontramos en una realidad dinámica donde el potencial de relación de las personas y de las cosas es más elevado que nunca; la cantidad de informaciones a las cuales tenemos acceso, sean estas acumuladas sea que se manipulen en tiempo real, es también más elevada que nunca; lo cual evidencia una gran cantidad de datos y objetos concebidos como elementos conectados, la gran velocidad con la cual se conectan y por lo tanto un elevado número de relaciones e interdependencias que se configuran y reconfiguran rápidamente y sin parar.
En este panorama, donde nuestra visión de la realidad es cada vez más dinámica y a la vez compuesta de conexiones, el objeto ya no se concibe como una entidad fija y aislada, sino más bien como parte integrante de un conjunto de relaciones que lo conforman, lo definen y lo conectan con lo demás.

Por otro lado, cabe recordar que la computación ha revolucionado el mundo del diseño en todas sus facetas y escalas; desde la introducción de los primeros softwares, fue claro que se trataba de herramientas que no sólo servirían para dibujar más rápido, sino que también definirían otras maneras de pensar, aquí también abarcando el entendimiento del existente y la ideación de nuevos artefactos, lo cual además de forjar y consolidar el concepto general del proyecto y de la forma como sistema, permite el conocimiento y el dominio de las geometrías complejas, desde su acepción teórica hasta su aplicación para la realización de objetos tangibles. En el camino hay un sinfín de herramientas, entre ellas la velocidad de cálculo, la capacidad de visualizar datos antes invisibles, la posibilidad de trabajar con algoritmos geométricos y visuales, bi y tridimensionales; donde uno de los puntos fuertes del dominio de dichas geometrías complejas es el manejo de los procesos de generación de la forma en cuanto estructura, con estudios que llevan a la formulación de reglas o patrones de organización cada vez más controlados y controlables.

De esta manera podemos bien decir que hoy en día, por un lado tenemos la capacidad de generar conformaciones complejas, cuya estructura procede de reglas de generación u organización bien definidas que determinan su naturaleza de manera muy clara, y que por otro lado, dichas conformaciones son sistemas de elementos y reglas que los relacionan, compuestos por partes fijas y otras variables, capaces de cambiar según parámetros conectados con el interior del sistema mismo o con el exterior, adaptándose y reconfigurándose según el tipo de dependencias, pero sin perder las bases de su propia identidad. 
Llevando el discurso al mundo del diseño, podemos ver cómo estas conformaciones tienen todas las características para traducirse en objetos, y por lo tanto objetos arquitectónicos, cuya estructura bien definida y cuya capacidad de adaptarse en relación con lo demás sin perder sus bases esenciales, los ponen al alcance de nuestro presente. Lo cual nos envía directamente a las características antes mencionadas de las geometrías complejas y por ende los objetos que potencialmente pueden surgir de las mismas: la capacidad de adaptarse según factores de variación, y al mismo tiempo la capacidad de volver a su estado inicial, por lo tanto, de poseer principios que le permitan de no perder su estructura de base.

A la luz de estas consideraciones, notamos como algunos conceptos más que otros se van consolidando como parte de nuestra forma mentis permeando en todas las capas de nuestras vidas, empezando desde como percibimos e interpretamos lo que nos rodea, hasta las maneras en que planteamos resolver problemáticas, satisfacer necesidades o elaborar nuevas ideas y artefactos.

Dentro de dichos conceptos, el de "resiliencia" aparece cada vez más y con diferentes matices, por aplicarse en varios campos de estudio, que van desde cuestiones tangibles como las propiedades de los materiales, a estudios sobre comportamientos y por lo tanto en el campo de la psicología o neurología, o en términos más sociales o urbanos, sólo por citar algunos; lo cual evidencia también la gran variedad de significados del término. Sin embargo, si buscamos el origen de la palabra encontramos que su raíz latina significa rebotar o sea volver atrás, que según la Real Academia Española se ha consolidado en dos acepciones: la primera como la capacidad de adaptación de un ser vivo frente a un agente perturbador o un estado o situación adversos, y la segunda como la capacidad de un material, mecanismo o sistema para recuperar su estado inicial cuando ha cesado la perturbación a la que había estado sometido.

Estas dos definiciones, que en realidad abarcan todas las otras variantes de la resiliencia, ponen el acento sobre dos puntos fundamentales de aquella visión de los objetos, y por ende de las formas y de las conformaciones, concebidos como sistemas de relaciones antes mencionada: la capacidad de adaptarse según factores de variación, y al mismo tiempo la capacidad de volver a su estado inicial, por lo tanto, de poseer principios básicos que le permitan de no perder su propia identidad.

Lo cual nos envía a los conceptos de adaptación de los seres vivos frente al contexto y al de sistema paramétrico.

Sobre la cuestión de adaptación de los seres vivos en respeto al contexto, según Jorge Wagensberg:

"Cualquier objeto real divide el mundo en dos partes: él mismo y el resto del mundo. (...) la relación del individuo con su entorno no se define con la idea de adaptación sino de independencia, un término que tiene un nítido significado a la hora de hablar de la estabilidad de un sistema y de sus eventuales interacciones con su mundo exterior. Un pedazo de materia inerte, por ejemplo, se calienta mansamente cuando en su entorno inmediato aumenta la temperatura. Se adapta. Un ser vivo, en cambio, tiende a reorganizarse para que su temperatura se mantenga como si en el entorno no hubiera ocurrido nada. Cambia para que nada cambie". (Wagensberg, 1989, p.42-43).

De donde, otra vez, se evidencia la importancia de los procesos de generación de las formas antes mencionados, sea en términos de definición de aquellas características que las identifican y delimitan en respeto al "otro", sea en cuanto a formulación de los tipos de relaciones con dicho "otro", al cual se adaptan con variaciones según reglas y rangos que son parte de la forma misma.

Lo cual nos lleva directamente al concepto de sistema paramétrico, y por ende al de diseño paramétrico, cada vez más común en las modalidades de elaboración de artefactos, sea en ámbitos profesionales que académicos. Un sistema paramétrico, en general, se compone de partes fijas y de partes variables, según las cuales definimos sea la naturaleza del sistema, o sea lo que nos dice "qué es el sistema" y lo que "no lo es", o sea "el otro"; y sus capacidades de permeabilidad o menos hacia dicho "otro", por las cuales el sistema se modifica mediante las variaciones de ciertos parámetros.

Los conceptos de variable y de parámetro, que contienen en sí un alto poder de reconfiguración y adaptación y a la vez la capacidad de volver a su estado inicial, son de gran impacto en nuestro presente, especialmente en el mundo del diseño, debido al desarrollo de softwares y herramientas que cada vez más favorecen la familiarización con la idea del proyecto como sistema y de adaptabilidad, interdependencia, relación etc. que se expresan aún más a con el diseño paramétricoasociativo y generativo en todas sus formas. Sin embargo, no se trata de algo nuevo, sino más bien tiene raíces muy lejanas. Si vamos más allá del campo del diseño y buscamos los orígenes del pensar paramétrico, encontramos que sus primeras manifestaciones conscientes se deben al concepto de algoritmo (Penrose,1989) y álgebra donde su gran diferencia con la aritmética es justo la de generar entidades capaces de variar aún sin perder su naturaleza; desde ejemplos muy sencillos como el de la diferencia entre la suma aritmética " $1+1=2$ " y su correspondiente algebraico " $a+b=c$ ", donde las letras no sólo pueden contener números sino también otras entidades que además de generar otros resultados, hasta podrían llegar a significar que $1+1$ es diferente a 2; pero donde el significado básico de suma, representado por ese "+", se mantiene como estructura fija, que nos garantiza que el sistema pueda volver a su estado inicial.

La diferencia entre álgebra y aritmética, trasladada desde el mundo de las matemáticas al de las formas, se convierte en un gran potencial de aquellos elementos capaces de adaptarse sin dejar de ser sí mismos, típica de los elementos generados a través del diseño paramétrico, y que hoy en día podemos considerar como propiedad imprescindible de las formas y de los objetos que se quieran relacionar con la complejidad de nuestro presente. El objeto se concibe, así como una estructura que nace de una serie de relaciones que lo conforman y a la vez definen sus variaciones, podemos hablar de la "resiliencia de los sistemas paramétricos", capaces de adaptarse, modificarse y volver al estado inicial.

En base a estas premisas nació la idea general de desarrollar una serie de morfologías generadas a través de sistemas paramétricos y estudiar sus formas de resiliencia. 
Siendo el concepto de "morfologías generadas a través de sistemas paramétricos" extremadamente genérico, mirando de manera más específica a aquellas procedentes de sistemas formales figurativos o menos (Chu, 2005), (Hofstadter, 2007) capaces de proliferarse por medio de componentes asociados, notamos que poseen un nivel más de resiliencia debido a la capacidad de los componentes de adaptarse recíprocamente; dando así origen a conformaciones reconfigurables que adquieren ciertas propiedades y características intrínsecas fuertemente vinculadas con esta resiliencia, que podemos llamar interna, ya que nacen de la misma y le proporcionan la capacidad de conectarse con el exterior.

Por otro lado, al considerar el pasaje de estas conformaciones desde el estado digital a su materialización física, sea como estudios de la forma finalizados a la generación de conocimientos, sea en cuanto objetos arquitectónicos reales, se vio oportuno también tomar en cuenta las formas de resiliencia de dichos sistemas a la hora de pasar desde lo digital a la materia. Por lo cual, desde la hipótesis de la existencia de varios tipos o niveles de resiliencia de las formas y conformaciones procedentes de sistemas paramétricoasociativos, el estudio se enfocó hacia el desarrollo de morfologías compuestas de proliferaciones de componentes, definidos por lenguajes formales, capaces de conformar geometrías complejas y el estudio de sus tipos de resiliencia.

Las morfologías desarrolladas se dividen en dos familias: morfologías abstractas y morfologías aplicadas; ambas con la intención general de desarrollar conocimiento sobre el potencial de resiliencia de las formas y de los sistemas paramétricos y así generar objetos más adecuados a nuestro presente; las primeras con un fin más teórico y las segundas con una aplicación real.

El trabajo se ha realizado en ámbito académico en la Universidad de Monterrey (UDEM) en México, como investigación del FabLab Monterrey aplicada a los cursos de Morfología, Geometrías Generativas y el estudio de Elementos de Arquitectura. Por lo cual, a los objetivos propios de la investigación, se juntan también los objetivos de cada uno de los cursos, relacionados con el aprendizaje y al fin de fomentar el dominio de los conceptos del pensar y el hacer avanzados desde las primeras etapas de la formación de los diseñadores.

En este paper se presentarán los resultados obtenidos hasta la fecha, evidenciando puntos fuertes y áreas de oportunidad.

\section{Procesos metodológicos}

Así como mencionado precedentemente, se desarrollaron dos tipos de morfologías: abstractas y aplicadas; tal división se debe más a las dinámicas de generación de las mismas que a su propia naturaleza, ya que aun si con algunas diferencias, todas se pueden definir como sistemas paramétricoasociativos que surgen de lenguajes formales; todas son conformaciones compuestas de proliferaciones de componentes.

En general el trabajo consiste en tres etapas fundamentales: siendo la primera el estudio y definición del componente y de sus variaciones, la segunda el estudio y definición de sus posibilidades de agregación y finalmente, la tercera el desarrollo de una configuración final que en algunos casos llega a su materialización física. Sin embargo, en este recorrido hay variaciones debidas a los tipos de estudios realizados.

En esta sección se presentarán las modalidades de desarrollo de dichas morfologías y los tipos de resiliencia individuadas como propias de sus sistemas.

\section{Morfologías M1: sistemas abstractos analógicos, desde el bottom-up al top-down.}

Este tipo de conformaciones se estudian dentro del curso de Morfología y consisten en el desarrollo de un sistema paramétrico-asociativo material, según los principios del form finding analógico y del bottom-up, para después realizar una conformación en top-down. Donde los objetivos principales del estudio son comprender y dominar geometrías complejas a través de las propiedades performativas de la materia.

El trabajo empieza con el estudio de un componente material, generalmente usando como base un cuadrado de foamy (Douglis, 2009), aplicando pliegues y cortes para explorar posibilidades de conformación dictadas por intuición y por las propiedades del mismo material; después de las primeras exploraciones se definen las reglas de configuración interna del componente, estableciendo así los primeros pasos de un sistema formal, y por lo tanto su control. Desde esta formulación, se estudian las posibilidades de variación que se exploran a través de materializaciones y a la vez se controlan como parte del sistema formal, estableciendo una familia de componentes y sus límites. Después se estudia la capacidad de agregación de los componentes, empezando a experimentar todas las agregaciones posibles entre dos elementos, para después pasar a uniones de más piezas. Cabe decir que en este proceso no hay una intención definida, sino más bien se trata de explorar las opciones dictadas por la naturaleza formal y material de los componentes; en seguida, se realizan sistemas unidimensionales con agregaciones lineales y sistemas di agregaciones bi y tridimensionales. Una vez desarrolladas dichas proliferaciones, se empiezan a analizar las características de los sistemas obtenidos, extrapolando las propiedades de cada uno, cuales elasticidad o rigidez, porosidad, cierre, compresión, expansión u otras que tienen más relación con la espacialidad, como por ejemplo la capacidad de envolver 0 contener etc. Posteriormente los estudiantes desarrollan un nuevo sistema, ahora sí con intención, para al final conformar una morfología que tenga una o más características espaciales 0 de habitabilidad bien definida: por ejemplo, generar una proliferación capaz de contener y que tenga tres niveles de porosidad, o generar un sistema envolvente auto-portante totalmente cerrado, etc., que, aun quedándose todavía a nivel de elementos abstractos, inician a acercarse al concepto de edificio.

Con estos estudios, los alumnos involucrados, familiarizan con el concepto de form finding, aprenden a observar las propiedades intrínsecas de la materia y de las formas y a formular lógicas de las formas dictadas por estas mismas; además entran en contacto por primera vez con el concepto de sistema emergente (Johnson, 2003), donde cada elemento tiene sentido al relacionarse con los demás, siendo capaz de 
adaptarse a varias condiciones según su propia naturaleza y los estados de los vecinos.

\section{Morfologías M2: sistemas abstractos digitales, top- down y sistemas formales}

Estos sistemas se estudian en el curso de Geometrías Generativas y consisten en el desarrollo de conformaciones complejas a través de la formulación de un lenguaje formal (Stiny,1980). Dichas conformaciones, contrariamente a las precedentes, nacen completamente en el entorno digital y se definen a través de la formulación de reglas previas. El trabajo se basa en el concepto de sistema visto como un conjunto de elementos y reglas los relacionan. Así como para las morfologías $\mathrm{M} 1$, se empieza con el desarrollo de un componente, esta vez en el entorno digital, formulado de manera precisa desde el inicio, a través de reglas establecidas que se expresan mediante sistemas de sustitución (Wolfram, 2002) o con sistemas geométricos sencillos. En ambos casos es fundamental la formulación de reglas bien formadas, que definen las bases del lenguaje formal. En este caso, antes se define un componente fijo indicando todos los pasos de su conformación; después siguiendo dichos pasos, se formula un algoritmo a través del cual se define el componente como entidad paramétrica, que llamamos "unidad algebráica" o también "axioma". Una vez establecido el componente con sus posibilidades y rangos de variaciones, se desarrolla su proliferación. Aquí se pueden seguir dos caminos; el primero es un proceso de crecimiento generativo, del tipo L-System, con reglas de desarrollo bien definidas, que serán los pasos sucesivos del lenguaje formal, donde dichas conformaciones se desenvuelven a varios niveles, formando en un inicio "letras", para después agregarse formando entidades más complejas que llamaremos "palabras", "frases" ... según el avance de las generaciones (Prusinkiewicz \& Lindenmayer, 2012). El segundo camino de crecimiento se basa en el hacer proliferar los componentes en entornos pre-establecidos, sean ellos mallas con diferentes tipos de organización y geometrías de base, o en superficies definidas, que pueden ser planas con varios niveles de curvatura y complejidad. En estos casos las reglas de agregación de los componentes están relacionadas con la naturaleza del entorno en el cual se desarrollan, que en general consisten en tipos de teselaciones triangulares, cuadrangulares, hexagonales, radiales, etc. En ambos casos, las proliferaciones, así como los componentes, son sistemas paramétricos-asociativos y se desarrollan en el entorno digital a través de algoritmos geométricos generados en Grasshopper.

Una vez definidas dichas conformaciones, donde las reglas de organización y los rangos de los parámetros pueden ser considerados como la estructura intrínseca del sistema, se introducen uno o más factores de influencia externos que afectan al sistema mismo. Dichos factores re-definen la naturaleza del sistema, que desde una entidad aislada se convierte en algo que comunica con el exterior y se modifica según criterios, también bien establecidos y formulados, que entran a hacer parte de su morfología, afectándola, pero sin minar la estructura de su definición formal inicial.

Uno de los puntos fundamentales de estos trabajos es el control: se trata de sistemas cuyas lógicas de la forma proceden de reglas bien definidas a priori, sean ellas procedentes de un conjunto de reglas generativas, sean ellas enmarcadas dentro de una forma existente que define la disposición de las piezas del sistema; manteniéndose en ambos casos a un nivel abstracto. Sin embargo en algunos pasos se empieza un acercamiento hacia problemáticas espaciales o condiciones de habitabilidad; por ejemplo en el caso de los sistemas que se desarrollan en superficies preestablecidas, se generan ciertas condiciones de envolvente, o al considerar un factor externo que afecta al sistema, se empieza a pensar en factores ambientales como la luz, el sol, o pre-existencias, como un camino o flujos de personas; con lo cual se inicia a dar un sentido más figurativo a dichos elementos de influencia y de consecuencia a las variaciones que provocan.

Estos sistemas, desarrollados completamente en el entorno digital, en algunos casos se materializan a través de la impresión 3D.

\section{Morfologías M3: sistemas aplicados}

Estas conformaciones se desarrollan a través de principios similares a las morfologías $\mathrm{M} 1$ y $\mathrm{M} 2$, pero con la gran diferencia que desde el inicio surgen con la intención de materializarse al fin de generar espacios habitables y que por lo tanto se desarrollan bajo una serie de limitantes y requisitos específicos.

Estos sistemas se realizan dentro del estudio de Elementos de la Arquitectura y son parte del proyecto Bichos, iniciado en el año 2013 y consolidado como línea de investigación del FabLab Monterrey en el año 2014, que fundamentado en los conceptos del "pensar y del hacer avanzados" (Frogheri \& Estévez, 2016) y en la introducción del diseño computacional y la fabricación digital desde las primeras etapas de la formación del diseñador, semestre tras semestre se concretiza en el diseño y la fabricación de pabellones a escala real.

Las morfologías de tipo M3 aquí presentes son los sistemas desde los cuales se generan las conformaciones de dichos pabellones y sus sistemas constructivos.

El proceso metodológico, como para las morfologías M1 y M2, desde la definición de un componente y sus posibilidades de variación, va al estudio de las posibilidades de proliferación y a la realización de una conformación final; además de completarse con su materialización, esta vez a escala real.

La diferencia es que en este caso los criterios a través de los cuales se conforman el componente y sus variaciones así como sus reglas de organización internas y las reglas de relación con los factores de influencia externos, dependen de las condicionantes del proyecto; las cuales se manifiestan de varias maneras, algunas con carácter espacial, otras estructurales y estáticas, otras más bien relacionadas con limitantes de tiempo, de espacio, de presupuesto económico o del feedback de las herramientas a través de las cuales se fabrican, sólo por citar algunas, así definiendo de esta manera el tamaño de los componentes, su número, el tamaño y la forma de la obra en general.

Aun así, tratándose de un sistema paramétrico- asociativo y generativo, formado como proliferación de componentes definidos a través de lenguajes formales, conserva las 
características de estos sistemas; así que por supuesto, siempre manteniéndose dentro del rango de las condicionantes indicadas, se desarrolla a través de procesos de form finding analógicos y digitales, en un inicio en modalidad bottom-up, dando origen a unos primeros sistemas analógicos, similares a las morfologías M1 pero finalizadas a generar los pabellones mencionados; desde los cuales es extraen las bases para el desarrollo de un sistema digital formulado a través de un lenguaje formal a varios niveles y con algoritmos geométricos según criterios de organización que proceden o de patrones establecidos como el panal, el close packing, la phyllotaxis etc. o de procesos generativos como L-system u otros tipos de fractales, así como las morfologías M2. Además, se considera la influencia de factores externos como elementos que afectan a la naturaleza del sistema mismo participando en su definición, siendo estos estos elementos externos en algunos casos factores ambientales o en otros hasta la presencia de personas.

Cabe subrayar que en este caso la materialización tiene un rol fundamental para el desarrollo de los sistemas, ya que, al concretarse en objetos arquitectónicos reales, en todas las etapas se realizan prototipos a escala real que retroalimentan el sistema y lo redefinen hasta llegar al resultado deseado. Así, podemos bien decir que las morfologías M3 juntan en sí las de M1 y las de M2 en sus procesos de morfogénesis, y que además contienen en sus reglas de conformación los requisitos de habitabilidad propios de un objeto arquitectónico real.

\section{Tipos de resiliencia: una primera propuesta de clasificación.}

Al desarrollar estas morfologías, se evidenciaron los siguientes tipos de resiliencia, basadas en las características propias de los sistemas que las definen y en sus capacidades de adaptarse al contexto. Cabe especificar que la clasificación aquí propuesta es una primera aproximación al tema y en su primera fase de formulación, por lo cual puede ser sujeta a cambios o redefiniciones en futuras etapas de la investigación. En seguida, se presentan los tipos de resiliencia individuados; algunas entre ellas se consideran exclusivas mientras otras se pueden combinar.

\section{Rs 1: Resiliencia singular de los componentes:}

Capacidad de variación de los componentes en cuanto entidades singulares y por lo tanto independiente a su relación con los demás, debida a factores de influencia; produce cambios dentro de los rangos de su naturaleza interna, y puede producir cambios de forma, de dimensiones, de resolución etc., que, desde una base inicial, puede generar familias de componentes.

Rs2: Resiliencia relacional de los componentes o resiliencia interna del sistema:

Capacidad de variación de los componentes, debida a la relación entre los componentes mismos entre ellos y a la asociatividad del sistema, que produce variaciones en ellos variaciones de forma, dimensiones, resolución etc., que, desde una base inicial, puede generar familias de componentes o elementos todos distintos de la misma familia. Se puede definir como resiliencia interna del sistema.

Rs3: Resiliencia general del sistema de componentes:
Capacidad de variación del sistema de componentes entendido como conjunto, debida a su asociatividad, causada por factores externos, que determina variaciones de forma, tamaño y dimensiones, número de elementos, resolución; la cual se puede considerar como una forma de adaptación a agentes externos. De este tipo de resiliencia puede surgir la conformación de un edificio relacionada con limitantes propias del proyecto, como por ejemplo cuestiones de posición, preexistencia, funciones o también en relación con factores logísticos de factibilidad, cuales tiempo, presupuesto económico, etc.

Además de los tres tipos de resiliencia individuados, que son vinculadas al concepto de sistema en cuanto tal, se individuaron otras dos categorías, que pueden ser combinadas con los tres precedentes de varias maneras: la resiliencia digital RD y la resiliencia material RM.

\section{$R D$ : La resiliencia digital:}

La resiliencia digital, puede ser de los tres tipos Rs1, Rs2, Rs3, pero existe solo en el entorno digital. Dicha resiliencia, en algunos casos se pierde a la hora de la materialización del sistema, mientras en otros casos puede permanecer y manifestarse también en el objeto tangible.

\section{RM: Resiliencia material:}

La resiliencia material es aquella que tiene que ver con el objeto material y puede manifestarse de diferentes maneras:

\section{RM1: Resiliencia intrínseca de la materia}

Es aquella capacidad de variación del componente o del sistema en cuanto conjunto, debida a las propiedades intrínsecas del material o de los materiales de los cuales se compone el sistema. Sean estas variaciones determinadas por las propiedades internas de relación entre elementos del sistema, o sean ellas debidas a la influencia de factores externos.

\section{RM2: Resiliencia material de la forma}

Es aquella capacidad del sistema, vinculada a su materialidad (material de los componentes y de sus uniones), de adaptarse a factores externos, cuales por ejemplo factores medio ambientales o sus usuarios, sólo por citar algunos, sin perder su identidad o sea manteniéndose intacta. Puede ser considerada también una resiliencia estructural, donde el objeto arquitectónico es capaz de auto-sostenerse y mantenerse firme.

Además, todas las formas de resiliencia material, pueden ser de tipo fijo o dinámico, donde las primeras son aquellas que una vez

\section{RMF: Resiliencia material fija}

Es la que poseen aquellas conformaciones que una vez materializadas, se quedan fijas o en el mismo estado.

\section{RMD: Resiliencia material dinámica}

Son aquellas conformaciones que una vez materializadas, son capaces de reconfigurarse a través de movimientos $u$ otros cambios de estado, debidos o a las propiedades intrínsecas de la materia o a la presencia de elementos sensibles y dispositivos electrónicos de entrada y salida en su sistema, 
que les permiten de recibir y elaborar datos, interactuando con el entorno.

\section{Resultados}

Por ser un trabajo vinculado con cursos y estudios de la Universidad, algunos de los cuales, desarrollados durante varios semestres, esta investigación se compone de una gran cantidad de sistemas. Por lo tanto, en esta sección se mostrarán algunas entre las más significativas de los tres tipos individuados en el apartado de procesos metodológicos y sus tipos de resiliencia.

\section{Morfologías M1}

Las morfologías de tipo M1 surgen desde estudios de form finding materiales fuertemente vinculados a la forma de las piezas, a su disposición, al material y al tipo de uniones; los sistemas que surgen (Figura1) tienen un alto nivel de asociatividad, por lo cual cualquier acción o influencia en un punto del sistema afecta a todo lo demás.

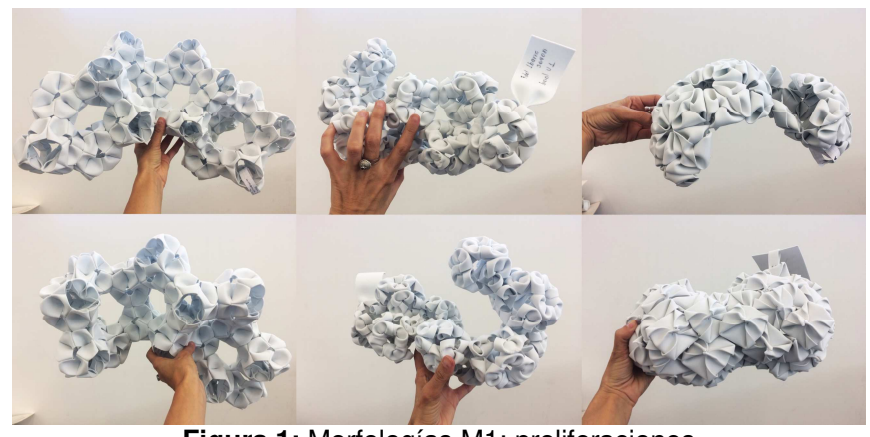

Figura 1: Morfologías M1: proliferaciones

En este tipo de sistemas se observaron las resiliencias de tipo R1, R2 y R3, además de las RM1 y RM3; En cuanto a la condición fija o dinámica de los sistemas, por la flexibilidad del material utilizado, en la mayoría de los sistemas se manifestó también la resiliencia dinámica RMD. Se trata de conformaciones que tienen una fuerte capacidad de deformarse y de volver a su estado inicial, por lo cual son muy útiles para entender el comportamiento de la materia y de los tipos de uniones entre elementos; sin embargo, muchas de las propiedades que poseen son debidas a la escala y a la flexibilidad del foamy utilizado; por lo tanto a la hora de quererlas traducir en objetos arquitectónicos reales casi siempre se tienen que re-geometrizar o congelar en uno de sus estados, pasando de la RMD a la RMF.

\section{Morfologías M2}

Las morfologías M2, sea que surgan de procesos generativos (Figura 2 y Figura 3), sea que proliferen en entornos preestablecidos (Figura 4 y Figura 5), poseen las resiliencias de tipo Rs1, Rs2, Rs3 y RD; sus materializaciones impresas en 3D poseen RM1 y RMF, aun si algunas, en los sistemas realizados, por las dimensiones y por el material de impresión, perdieron esta última, rompiéndose o deteriorándose (Figuras $6,7,8$ y 9$)$.

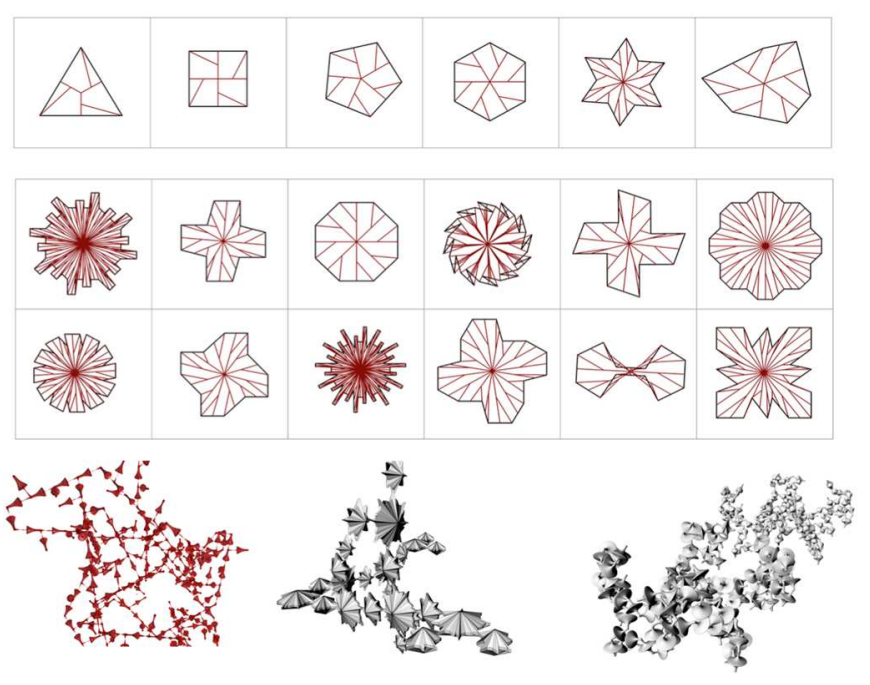

Figura 2: Morfologías M2 generadas con LSystem



Figura 3: Impresión 3D de M2 generadas con LSystem

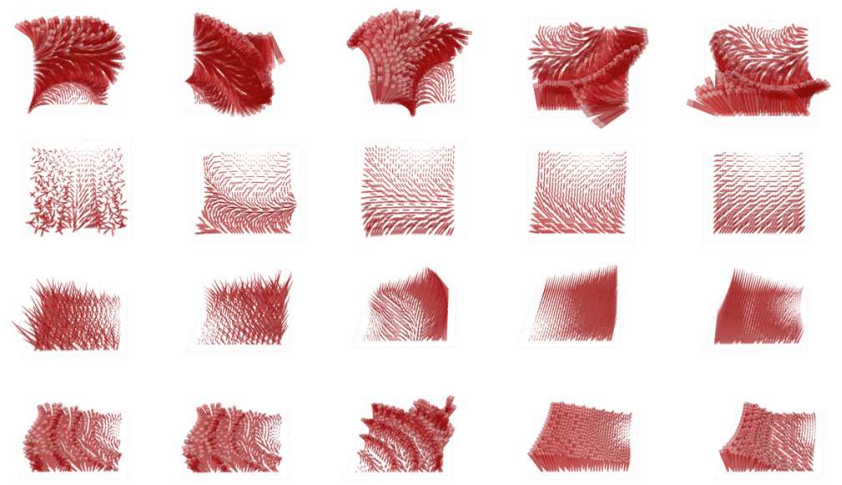

Figura 4: Morfologías M2 proliferadas en teselaciones

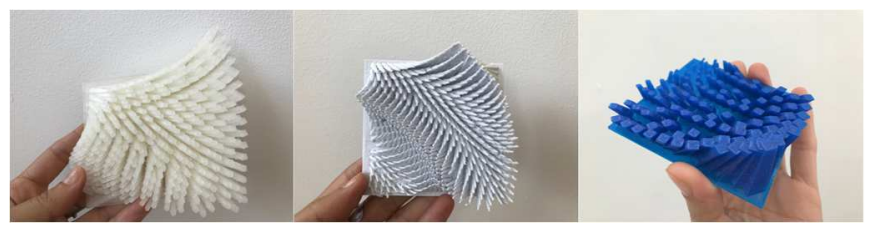

Figura 5: Impresión 3D de M2 proliferadas en teselaciones 

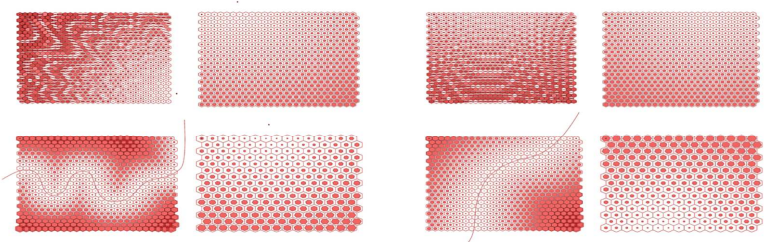

Figura 6: Morfologías M2 proliferadas en mallas
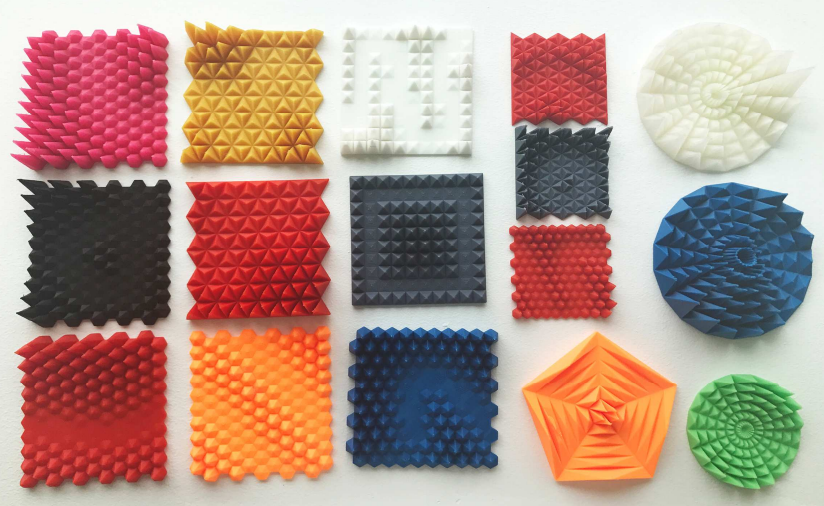

Figura 7: Morfologías M2 proliferadas en mallas

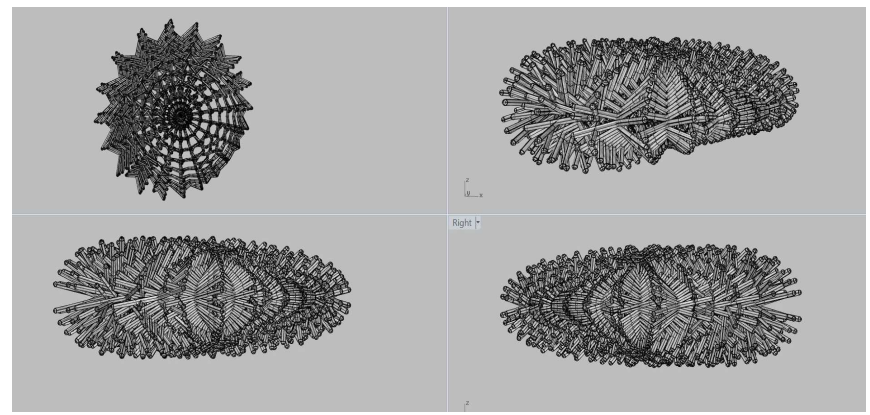

Figura 8: Morfologías M2 proliferadas en superficies

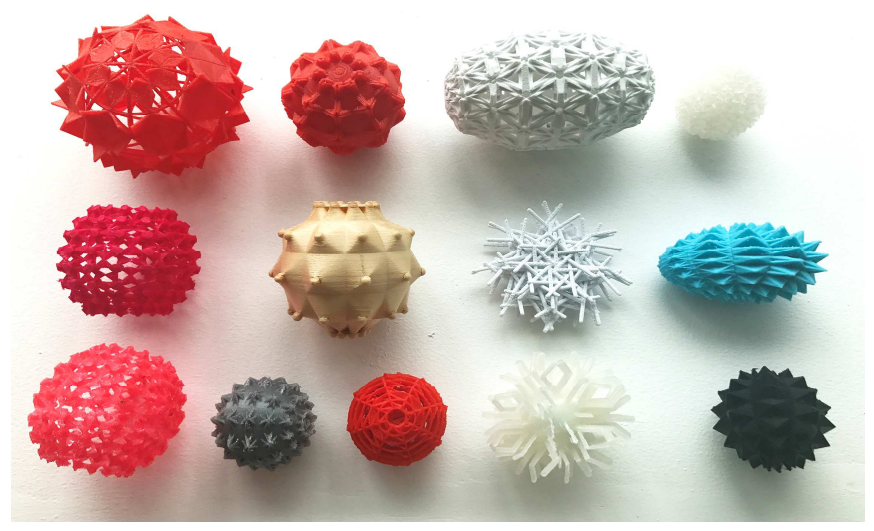

Figura 9: Impresión 3D de M2 proliferadas en superficies

\section{Morfologías M3}

Las Morfologías M3 son las que dieron origen a los pabellones de la familia Bichos (Figura 10).

Dichas estructuras poseen las resiliencias de tipo Rs1, Rs2, Rs3 y la RD en su fase de desarrollo digital (Figuras 11 y 12) y en general las resiliencias materiales RM1, RM2 y RMF.
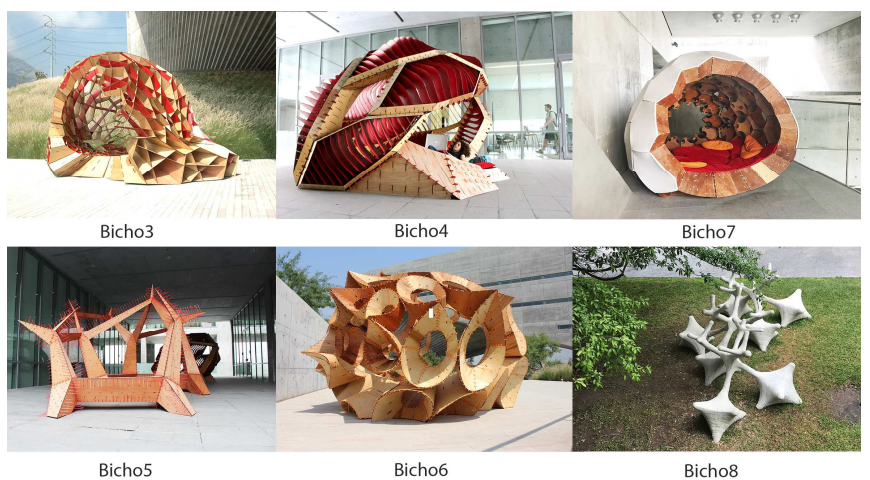

Bicho7

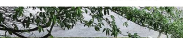

Bicho5

Bicho6

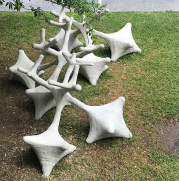

Bicho8

Figura 10: Morfologías M3: Pabellones de la familia "Bichos"

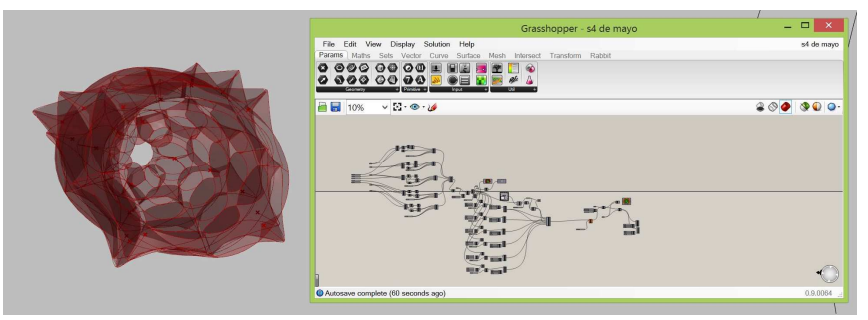

Figura 11: Morfologías M3: sistema digital del bicho6


Figura 12: Morfologías M3: Rs3 y RD del Bicho6 en fase digital



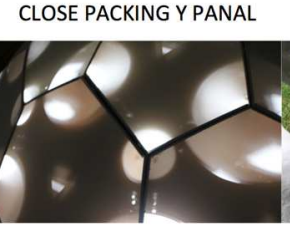

CLOSE PACKING Y PANAL
PHYLLOTAXIS

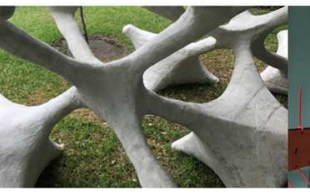

L-SYSTEM
TESELACIONES



L-SYSTEM
Figura 13: Morfologías M3: criterios de organización 
Entre ellas, el pabellón Bicho7, sensible gracias a sensores e dispositivos electrónicos, reacciona a la presencia de personas con cambios de forma y de colores, por lo cual posee también la resiliecia RMD (Figura 14).

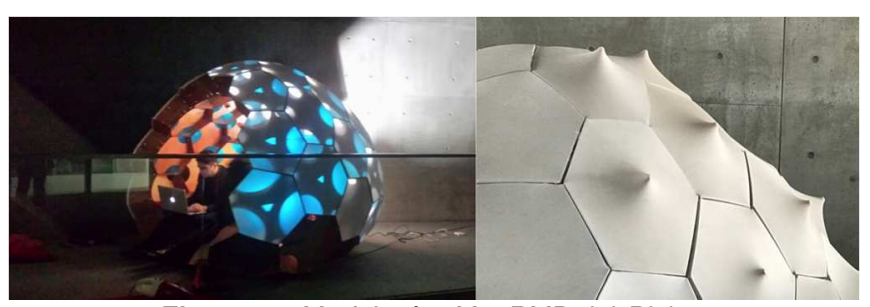

Figura 14: Morfologías M3: RMD del Bicho7

\section{Conclusiones}

Uno de los retos fundamentales del trabajo aquí presentado fue vincular el concepto de resiliencia en su sentido más básico a las propiedades de los sistemas paramétricos asociativos y al de adaptación de los seres vivos; desde lo cual surgen algunos puntos a considerar sea como argumentos de reflexión para nuevas etapas de la investigación, sea ya como factores para el desarrollo de conformaciones y objetos arquitectónicos capaces de estar al paso con nuestro presente.

El primero es que, si es verdad que cada vez más interpretamos lo existente y elaboramos nuestros artefactos tomando en cuenta la capacidad de conexión de las cosas y su capacidad de adaptación, también es verdad que en el campo del diseño solemos pensar en dicha adaptación de manera más parecida a las propiedades de los materiales inertes (Wagensberg, 1999), que a la que caracteriza los seres vivos, considerando más el potencial de variación que su capacidad de mantener su identidad. Sin embargo, los objetos, así como los concebimos hoy en día, por ser sistemas y gracias a los alcances de la tecnología, conceptualmente tienden cada vez más a parecerse a organismos que a simples pedazos de materia; lo cual es fundamental para poderse relacionar con las características y las exigencias de las problemáticas del presente.

Por otro lado, está la cuestión del pasaje desde lo digital a la materia: solemos dominar los sistemas paramétricos en el entorno digital, pero a la hora de materializarlos se vuelven casi todos fijos y congelados en un estado, que es sólo uno de los infinitos posibles de los que puede generar el sistema; lo cual como hemos visto posee cierto tipo de resiliencia, pero casi nunca aquel dinamismo necesario para estar al alcance de las problemáticas espaciales que pretende resolver.
Otro punto consiste en evidenciar la riqueza del concepto de resiliencia y la manera en que se puede manifestar en los sistemas desarrollados, sobre todo en su vinculación con la forma y con sus propiedades intrínsecas, cosa que en general no se suele estudiar de esta manera, sino con un enfoque más dirigido al potencial de variación en cuanto tal; lo cual nos muestra una valiosa aplicación de nuestra actual capacidad de dominar los procesos de generación de la forma, que quizás todavía no estamos aprovechando en pleno.

Por último, cabe subrayar que el siguiente trabajo es la primera etapa de una investigación donde se exploraron sólo determinados tipos de sistemas y se planteó una primera propuesta de clasificación de sus tipos de resiliencia; lo cual, en parte por ser una primera aproximación al tema y en parte debido a su carácter taxonómico, es susceptible a reajustes y reformulaciones; por lo tanto, se planea seguir con la investigación, con nuevos avances y evoluciones en sus desarrollos futuros.

\section{Referencias}

Chu, K. (2005). Metafísica de la arquitectura genética y computación. Barcelona, España: ESARQ.

Douglis, E. (2009). Autogenic structures. New York: Taylor \& Francis.

Frogheri, D. \& Estévez, A.T., (2016) Entre el pensar y el hacer avanzados, pp. 219-226, en XX Congreso de la Sociedad Iberoamericana de Gráfica Digital [Blucher Design Proceedings, v. 3, n. 1], Blucher, São Paulo.

Hofstadter, D.R. (2007). Godel, Escher, Bach: un eterno grácil bucle, Tusquets Editores

Johnson, S. (2003). Sistemas emergentes: o qué tienen en común hormigas, neuronas, ciudades y software. Madrid: Turner.

Prusinkiewicz, P., \& Lindenmayer, A. (2012). The Algorithmic Beauty of Plants. Springer Science \& Business Media.

Stiny, G. (1980). Introduction to shape and shape grammars, in Environment and Planning B, 1980, volume7, pages 324-351.

Wagensberg, J. (1999, mayo) Complejidad e Incertidumbre. Mundo Científico (201)

Wagensberg, J. (2004) "La rebelión de las formas o cómo preservar cuando la incertidumbre aprieta" $1^{\text {a }}$ edición. España. Tusquets Editores, Metatemas MT8

Wolfram, S. (2002)"A New Kind of Science", First edition. Champaign, IL, USA: Wolfram Media Inc.

Wolfram, S. (1984). Cellular automata as models of complexity. Nature, 311(5985), 419-424. https://doi.org/10.1038/311419a0 